Tani Sagna*, Elena Bonora, Marie Nabonswindé Lamoussa Ouedraogo, Daniela Fusco, Abdou Azaque Zoure, Cyrille Bisseye, Florencia Djigma, Jacques Gilbert Kafando, Nayi Zongo, Zoenabo Douamba, Dorcas Obiri-Yeboah, Daniela Turchetti, Virginio Pietra, Olga Melanie Lompo, Charlemagne Ouedraogo, Marco Seri, Jacques Simpore

\title{
Identification of BRCA1/2 p.Ser1613Gly, p.Pro871Leu, p.Lys1183Arg, p.Glu1038Gly, p.Ser1140Gly, p.Ala2466Val, p.His2440Arg variants in women under 45 years old with breast nodules suspected of having breast cancer in Burkina Faso
}

https://doi.org/10.1515/bmc-2019-0015

received February 5, 2019; accepted May 7, 2019.

Abstract: Breast cancer is the top cause of cancer mortality among women in the world and the second in Africa. The aims of this study were to: i) identify women with breast nodules suspected of having breast cancer ii) sequence the $B R C A 1$ and BRCA2 genes and iii) screen mutations. From

*Corresponding author: Tani Sagna, Centre de Recherche Biomoléculaire Pietro Annigoni (CERBA)/LABIOGENE, Université de Ouagadougou, 01 BP 364, Burkina Faso; Institut de Recherche en Sciences de la Santé (IRSS), Ouagadougou, 03 BP 7192, Burkina Faso, E-mail: stanilinda@gmail.com

Marie Nabonswindé Lamoussa Ouedraogo, Abdou Azaque Zoure, Cyrille Bisseye, Florencia Djigma, Zoenabo Douamba, Virginio Pietra, Jacques Simpore: Centre de Recherche Biomoléculaire Pietro Annigoni (CERBA)/LABIOGENE, Université de Ouagadougou, 01 BP 364, Burkina Faso.

Elena Bonora, Daniela Fusco, Daniela Turchetti, Marco Seri: U.O. Genetica Medica Dipartimento di Scienze Ginecologiche, Ostetriche, Pediatriche, Policlinico S. Orsola-Malpighi, Bologna, 902 D 40138, Italy.

Abdou Azaque Zoure: Institut de Recherche en Sciences de la Santé (IRSS), Ouagadougou, 03 BP 7192, Burkina Faso.

Virginio Pietra, Jacques Simpore: Hôpital Saint Camille,

Ouagadougou, 09 BP 444, Burkina Faso.

Jacques Gilbert Kafando, Nayi Zongo, Olga Melanie Lompo, Charlemagne Ouedraogo: Centre Hospitalier Universitaire Yalgado Ouedraogo (CHU/YO), Ouagadougou, 03 BP 7022 Burkina Faso. Dorcas Obiri-Yeboah: Department of Microbiology and Immunology, School of Medical Sciences, University of Cape Coast, P. O. Box 5007, Ghana.

Cyrille Bisseye: Département de Biologie, Faculté des Sciences Université des Sciences et Techniques de Masuku (USTM), BP 934 Gabon.
2015 to 2016, 112 women aged from 35 to 44 years, who had come for consultation in the gynecology/obstetrics and the oncology department of the University Hospital Yalgado Ouedraogo, voluntarily agreed to participate to this study. Whole blood was collected from those with mammary nodules. The genomic DNA was extracted using Qiagen kit. FAST KAPA was used for genomic DNA amplification and the purified PCR products were analyzed by direct sequencing using Big Dye v1.1 and ABI 3730 automated sequencer. Nucleotides substitutions were determined. We identified BRCA1 SNPs rs1799966, rs799917, rs16942, rs16941, rs2227945, and BRCA2 SNPs rs169547, rs4986860. These identified variants are found mostly in cases of benign tumors of breast or ovarian cancer with familial history of breast cancer. This study in Burkina-Faso, is the basis for improved and more specific genetic testing, and suggests that additional genes contributing to an increased risk of breast cancer should be analyzed.

Keywords: BRCA; Breast cancer; Burkina Faso; SNPs; Polymorphism.

\section{Introduction}

Breast cancer is the most common cancer in women, especially in those over 50 years old for whom it is the leading cause of death worldwide. The global trend in the epidemiology of breast cancer in African countries is not far from that observed in the western countries of the world. Breast cancer is the second most frequent cancer both in the totality of African countries and in the

¿ Open Access. (C) 2019 Tani Sagna et al., published by De Gruyter. (cc)BY This work is licensed under the Creative Commons Attribution-NonCommercial NoDerivatives 4.0 License. 
specific area of Western Africa (which comprises Burkina Faso) accounting for the 25 to $30 \%$ of cases of cancers [1]. Nevertheless, its incidence is higher in developed than in developing countries $(64.2 / 100,00$ vs $23.1 / 100,000$ women) [2].

Breast cancer is a malignant tumor of the mammary gland. This cancer is most common in women, with 89 cases per $100,000.5$ to $10 \%$ of these cancers have a clear genetic basis, whereas $85-90 \%$ of cases (called sporadic or non-hereditary) have a complex etiology still poorly understood [3-6]. In Burkina Faso (one of the west African countries) breast cancer is being responsible for $17.7 \%$ of women's deaths [7]. A recent study provide preliminary information about breast cancer risk decreasing with multiparity among women in Burkina Faso [8, 9]. Genetic and non-genetic factors are involved in the etiology of breast cancer.

The genetics of breast cancer in African countries is generally undetermined. A genetic test may reveal the risk to women from a family where there is a history of mutations in a specific gene $[10,11]$. The lack of data on the genetics of breast cancer in African countries might be due to lack of diagnostic tools in molecular biology, preventing women from performing this test [2, 12-15]. Two predisposing genes for breast cancer have been identified [16, 17] so far: BRCA1 on chromosome 17 (more than 500 mutations or sequence variations have been identified) and BRCA2 on chromosome 13 (over 100 different mutations have been reported). Women who have inherited mutations in BRCA1 (17q21, chromosome 17: base pairs 43,044,294 to 43,125,482) or BRCA2 (13q12.3, chromosome 13: base pairs $32,315,479$ to $32,399,671$ ) have an increased risk of developing breast and ovarian cancers $[11,17]$. The probability of developing breast cancer in a carrier of a BRCA1 mutation is about $65 \%$ before the age of 70 and $45 \%$ for carriers of mutations in the BRCA2 [3, 18, 19]\{Couch, 1997 \#2200;Whittemore, 1997 \#2219;Mote, 2004 \#2212;Whittemore, 1997 \#2219\}. In a study by Rebbeck et al, among BRCA1 mutation carriers, 46\% of women were diagnosed with breast cancer, $12 \%$ with ovarian cancer, $5 \%$ with breast and ovarian cancer, and $37 \%$ without cancer. And among BRCA2 mutation carriers, $52 \%$ of women were diagnosed with breast cancer, $6 \%$ with ovarian cancer, $2 \%$ with breast and ovarian cancer, and $40 \%$ without cancer [20].

The available information about breast cancer in Burkina Faso, regarding the histo-pathological characteristics of tumors and parity risk factors, is limited [8,9], with few specific records about genetic features $[8,9,21]$.

The aim of this study was to provide the first ever cancer related genetic information for a West African country. To achieve this aim, these specific objectives were targeted: i) identify women with breast nodules suspected of being breast cancer; ii) determine genetic variants associated with breast cancer in Burkina Faso by PCR.

\section{Materials and methods}

\section{Study population}

This study took place from June 2015 to May 2016 and was approved by the Ethics Committee for Health Research of Burkina Faso ( $\mathrm{N}^{\circ}$ 2014-8-098). The eligibility criteria were i) patients with diagnosis of breast cancer before age 45; ii) patients able to provide informed consent to complete detailed epidemiological questionnaire, which includes details of family history of cancer; and iii) patients provided a blood sample for genetic analysis. After obtaining written informed consent from each women, aged from 35 to 44 years old (with an average of 40.00 \pm 3.04) with breast nodules suspected of having breast cancer, blood specimens were collected from the General Surgery and Gynecology-Obstetrics services and oncology department of the University Hospital Yalgado Ouedraogo.

Ethical approval: The research related to human use has been complied with all the relevant national regulations, institutional policies and in accordance the tenets of the Helsinki Declaration, and has been approved by the Ethics Committee for Health Research of Burkina Faso ( $\mathrm{N}^{\circ}$ 2014-8-098).

Informed consent: Informed consent has been obtained from all individuals included in this study.

\section{$B R C A 1$ and $B R C A 2$ mutation screening}

The genomic DNA was extracted from blood specimens with a Qiagen kit at the Biomolecular Research Center Pietro Annigoni (CERBA) of Ouagadougou, Burkina Faso. The remaining steps for the genetic analysis of DNA were performed at the St'Orsola-Malpighi Hospital, Laboratory of Medical genetics, University of Bologna, Italy. The entire coding sequence and part of the regulatory sequence of BRCA1 and BRCA2 were amplified by PCR using Fast KAPA Master Mix 2x with the following cycling protocol: $95^{\circ} \mathrm{C}$ $1 \mathrm{~min}(1 \mathrm{step}) ; 95^{\circ} \mathrm{C} 15 \mathrm{~s}, 58^{\circ} \mathrm{C} 15 \mathrm{~s}, 72^{\circ} \mathrm{C} 15 \mathrm{~s}$ (40 cycles); $72^{\circ} \mathrm{C} 15 \mathrm{~s}$ (1 step). Sequences were resuspended in Injection Buffer (10 $\mu \mathrm{L}$, Millipore) and loaded onto the ABI 3730 automated sequencer (Thermofisher Scientific, Foster City, CA). 


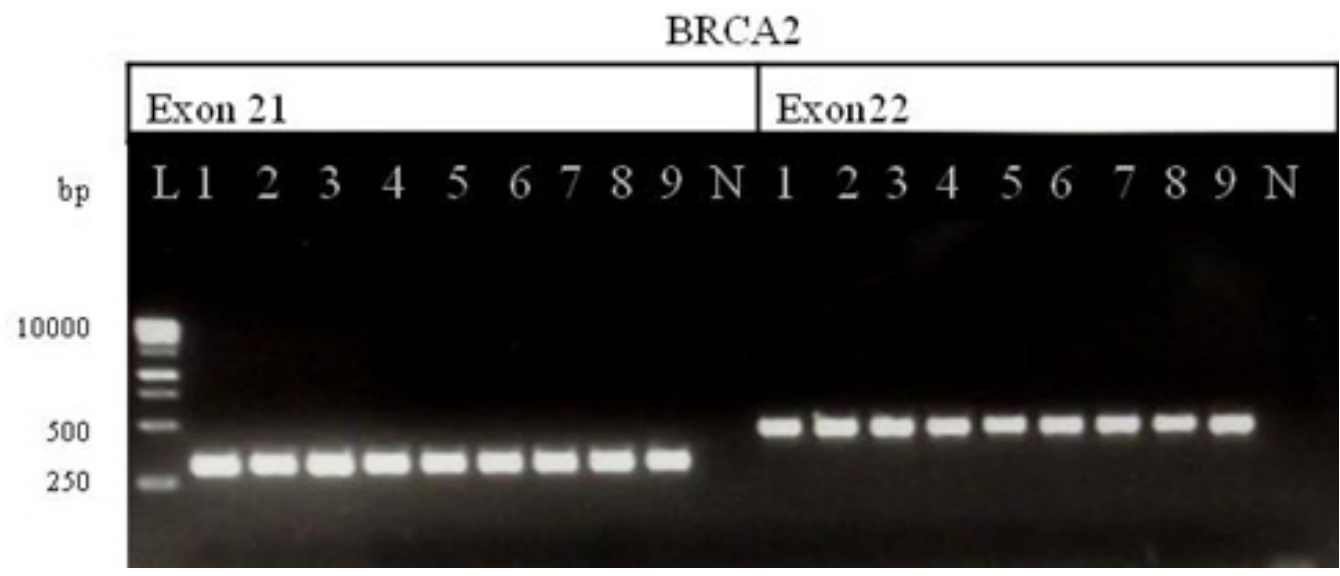

Figure 1: $B R C A 2$, Exon 21 and 22 PCR product profile in agar gel 2\%. Legend: L: Fermentas GeneRulerTM DNA Ladder \#SM0313; N: Negative control; Number 1 to 9: specimens.

\section{Sequence analysis}

Data from the ABI3730 sequencing machine were analyzed using the software Chromas lite v2.5. We compared the sequences with the reference sequence reported in "Ensembl genome" browser and the variants were classified by comparison using dbSNP and ClinVar.

\section{Results}

\section{Patient's characteristics}

From 2015 to 2016, 112 women aged from 35 to 44 (average of $40.00 \pm 3.04$ years), who had come for consultation in the service of General Surgery and Gynecology-Obstetrics services and the oncology department of the University Hospital Yalgado Ouedraogo, voluntarily agreed to participate in this study. Based on the eligibility criteria, 9 women were screened for genetic breast cancer. Of the 9 eligible patients, 2 had been diagnosed with cancer before the age of 36 and 2 other patients had a family history of cancer ( 1 breast cancer and 1 foot cancer) and all were at the level T4 of TNM.

\section{Mutation screening}

After loading the PCR products on $2 \%$ agarose gel $(3 \mathrm{~mL})$ they were visualized under UV light as reported in Fig 1 (examples of BRCA1 and BRCA2 profiles). An example of the sequencing profile of an heterozygous specimen is reported in Fig 2 in exon 10 of BRCA1: a non-synonymous and heterozygous change at the $6^{\text {th }}$ base pair is present

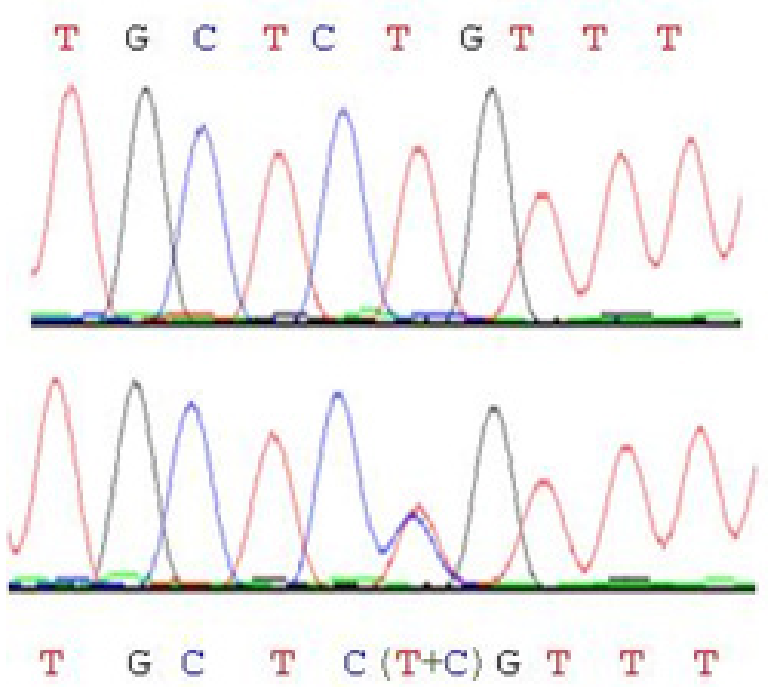

Figure 2: Sequencing profile of a DNA. Legend: variation heterozygous change at the 6 th base pair $(T / T+C)$.

in the figure corresponding to the change c.2622 $>\mathrm{T}$; p.Leu871Pro, rs799917), where a clear double peak of C/T could be identified in the lower panel. The upper panels shows a non-synonymous change but in homozygous state. All specimens had a non-synonymous change at this site.

\section{Variants}

All women in this study had at least one genetic variation, such as synonymous or non-synonymous amino acid variation. Some also had intron variations. In specimen 1 , which had more genetic variation than other specimens, we identified a 3'UTR change in the last exon of BRCA1 


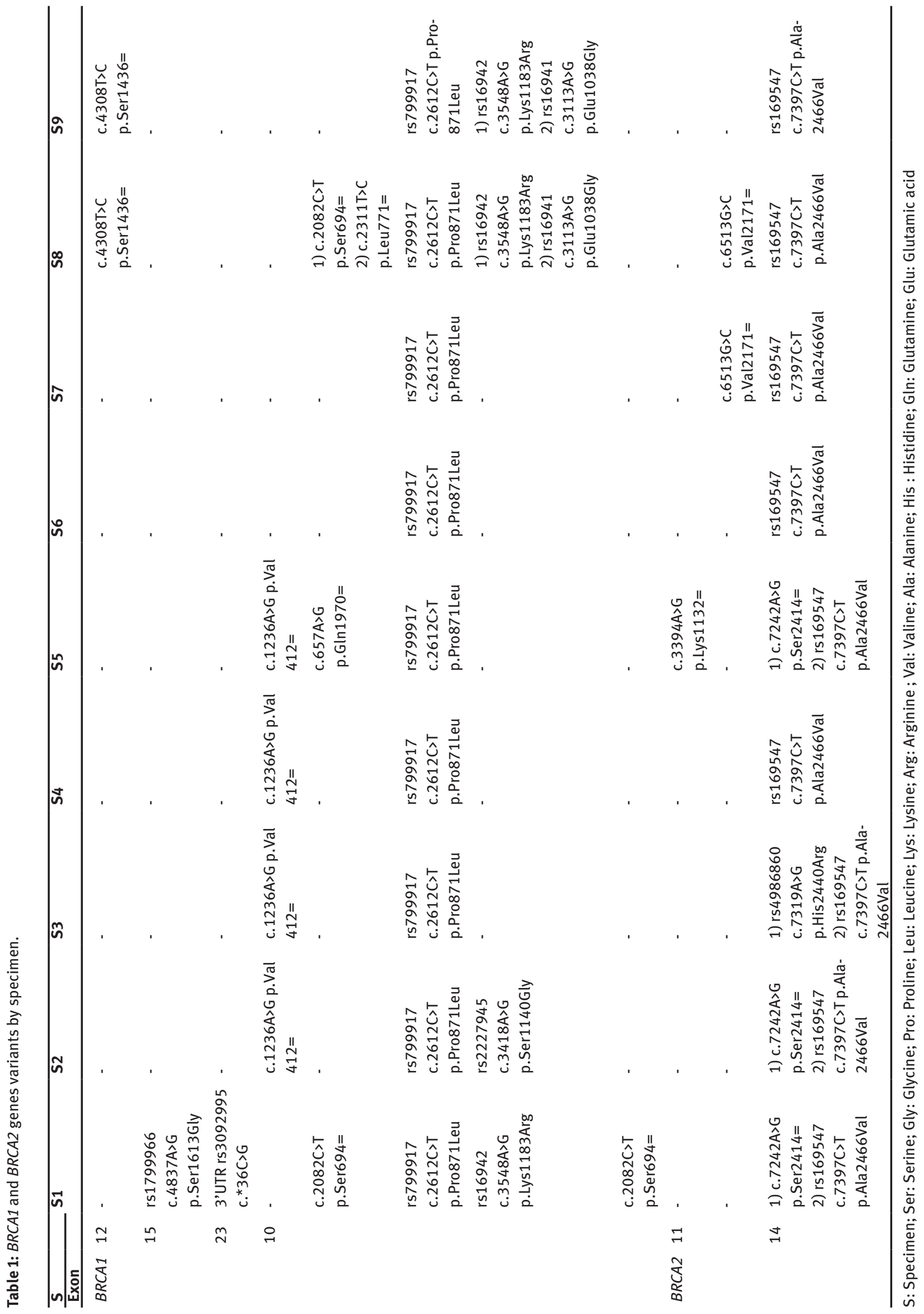


Table 2: List of known BRCA1 SNPs identified in this study and minor allele frequencies compared with other populations.

\begin{tabular}{|c|c|c|c|c|c|c|c|c|c|c|c|}
\hline Gene & SNP & $\begin{array}{l}\text { Amino acid } \\
\text { change }\end{array}$ & $\begin{array}{l}\text { Protein } \\
\text { change }\end{array}$ & $\begin{array}{l}\text { Chrs:bp } \\
\text { dbSNP }\end{array}$ & $\begin{array}{l}\text { Allele } \\
\text { Frequency }\end{array}$ & Frequency* & ASW & ESN & YRI & AFR & EUR \\
\hline \multirow[t]{5}{*}{$B R C A 1$} & rs1799966 & c. $4837 A>G$ & p.Ser1613Gly & $17: 43071077$ & A/G $0.356(G)$ & $0.111(1 / 9)$ & 0.246 & 0.172 & 0.194 & 0.225 & 0.360 \\
\hline & rs799917 & c. $2612 C>T$ & p.Pro871Leu & $17: 43092919$ & $\mathrm{C} / \mathrm{T} 0.544(\mathrm{~T})$ & $0.889(8 / 9)$ & 0.262 & 0.944 & 0.074 & 0.885 & 0.738 \\
\hline & rs16942 & c. $3548 \mathrm{~A}>\mathrm{G}$ & p.Lys1183Arg & 17:43091983 & A/G $0.353(G)$ & $0.333(3 / 9)$ & 0.246 & 0.167 & 0.181 & 0.218 & 0.355 \\
\hline & rs16941 & c. $3113 A>G$ & p.Glu1038Gly & $17: 43092418$ & A/G $0.336(G)$ & $0.222(2 / 9)$ & 0.148 & 0.126 & 0.122 & 0.157 & 0.357 \\
\hline & rs2227945 & c. $3418 \mathrm{~A}>\mathrm{G}$ & p.Ser1140Gly & $17: 43092113$ & A/G $0.010(\mathrm{G})$ & $0.111(1 / 9)$ & 0.009 & 0.056 & 0.023 & 0.036 & 0.001 \\
\hline \multirow[t]{2}{*}{$B R C A 2$} & rs169547 & c. $7397 C>T$ & p.Ala2466Val & $13: 32355250$ & $\mathrm{C} / \mathrm{T} 0.976(\mathrm{~T})$ & $1(9 / 9)$ & 0.918 & 0.944 & 0.907 & 0.911 & 0.999 \\
\hline & rs4986860 & c.7319A>G & p.His2440Arg & $13: 32355172$ & $A / G 0.010(G)$ & $0.111(1 / 9)$ & 0.033 & 0.045 & 0.014 & 0.039 & 0 \\
\hline
\end{tabular}

$p$-value between muted minor allele frequency in our specimens and in Yoruban population (YRI): $p=0.409$; Chrs: chromosome; *: allele frequency in our specimens;

Population description: ASW: African ancestry in Southwest USA; ESN: Esan in Nigeria; Yoruban in Ibadan (Nigeria); AFR: African; EUR: European.

(exon 23). We identified several common polymorphisms in both BRCA1 and BRCA2 in our specimens (Table 1). Nonsynonymous coding changes are represented with the SNP identity. The others with no SNP identity are synonymous coding.

There are more variant alleles in our population for $B R C A 1$ than for $B R C A 2$ (Table 2). For BRCA1 we identified: A/G (c.4837A $>$ G rs1799966; c.3548A $>$ G rs16942; c.3113A $>$ G rs16941; c.3418A $>\mathrm{G}$ rs2227945), C/T (c.2612C $>\mathrm{T}$ rs799917); and for BRCA2 A/G (c.7319A $>$ G rs4986860), C/T (c.7397C >T rs169547). All of these variants are missense mutations with benign clinical significance. Based on physical and comparative consideration these missense variants are not likely to affect protein function (ClinVar). There is no statistical significant difference in the frequency of the alleles between ours population group and Yoruban population ( $\mathrm{p}>0.05)$. The SNP rs169547 (BRCA2 non synonymous variation c.7397C $>\mathrm{T}$; p.Ala2466Val) was found in all women of our study, however we found the proportion to be statistically similar to both European and most of others populations ( $p>0.05$ ) (Table 2).

\section{Discussion}

$B R C A 1$ and BRCA2 genes have been screened in young women with nodules, suspected of being breast cancer, and some variants have been found. Each specimen in this study had at least one variant. All variants identified are benign. However, others populations have high proportions of oncogenic variants. For example, in the case of Ashkenazi families, a high frequency of causative mutations of the BRCA1 and BRCA2 genes, as insertions and deletions, were observed [22, 23]. A single variation 3'UTR was found in the last exon (BRCA1 exon 23) with reported SNP rs3092995, it is possible that this variation may alter the mRNA structure in this specimen [24, 25]. All identified variants were located between c. $2612 \mathrm{C}>\mathrm{T}$ and c. $4837 \mathrm{~A}>\mathrm{G}$ regions for $B R C A 1$ and between c.7319A $>\mathrm{G}$ and c.7397C $>\mathrm{T}$ regions for $B R C A 2$. These regions are not located in the breast cancer cluster regions identified by Rebbeck and Collaborators in 2015, but some of these are in the ovarian cancer cluster region (Exon 11 of BRCA1) [20]. This revealed that breast cancer may have nongenetic predictor factors as well as varied genetic factors. The variants identified in this study are found mostly in cases of benign tumors. According to a study published in 2008, non-cancerous breast diseases may promote the subsequent occurrence of cancer [26]. Some of the variants we identified may be predisposing factors. In general, variants may not cause cancer. Some variants, oncogenic in other countries may not be in Africa because the genotype may be influenced by the environment [27]. Some mutations can act synergistically to cause cancer [28], especially since in this study there were patients with family history of cancer.

Among our study population, we identified the same variation (regarding the amino acid change c.2622C>T corresponding to the protein change p.Leu871Pro) that like other variations found in our specimens, had been reported previously in dbSNP database. This common variation is on the BRCA1 gene (SNP rs799917). Some 
studies have found that BRCA1 rs799917 polymorphism may contribute to the risk of cervical cancer in Chinese populations, and further validation in other populations would be warranted [29]. Recently in Burkina Faso, Zoure et al. targeted exon2, exon 5 and exon11A for BRCA1 sequencing [21]. These exons were described as likely to contain causative mutations of cancer in northern Africa and some west African countries' $[30,31]$. But they did not detected mutations in any of the 15 women diagnosed with family breast cancer history in Burkina Faso [21]. In our study, we sequenced the whole BRCA1 and BRCA2 gene. This is the first reported study which screened these genes in their entirety to detect mutations in a Burkina Faso population. It is worth noting that our specimen size was very small and selected mostly based on the age of presentation. Also, in the familial cases of breast cancer, the proportion of $B R C A 1$ and $B R C A 2$ carrier is relatively low $(5 \%)[3,4,32]$ and this could explain why known oncogenic variants have not yet been determined that confer an increased risk for breast cancer in this study.

Indeed few studies concerning BRCA1 and BRCA2 in the West African population have been published. We can consider the study in Yoruban populations where the BRCA1 Y101X mutation (1.1\%) has been identified [33]. A study carried out in Algeria observed that $9 \%$ of sporadic cases of early-onset breast cancer and 36\% of family cases have been associated with BRCA1 mutations [34]. In Ghana another high proportion (61\%) of mutation was found among patients with breast cancer [35]. Zeng and collaborators performed a study involving Africans, in which no association between identified SNPs and breast cancer risk was identified [36]. The limited data may indicate that the west African families do not have the same access to screening for mutations in target genes compared to other countries [22, 23]. The detection of $B R C A$ mutations within a family member would have predicted hereditary breast cancer [3, 18, 19]. For those at risk, also for other family members, active surveillance through early detection would be necessary. In addition, mutations may vary among individual racial and ethnic groups [22, 37] and early studies suggested that BRCA mutations were relatively rare among Africans $[14,33]$.

The variant c.4837A $>\mathrm{G}$ (corresponding to the protein substitution p.Ser1613Gly) has been found to be most frequent in South Asian (50\%), East Asian (37\%), European (36\%), American (37\%) and Africans (23\%) populations'. About $77 \%$ of the alleles found in the African population studies are ancestral allele $\mathrm{T}$ (dbSNP). This proportion is similar to those found in the African sub-populations with variant c.2612C >T rs799917 SNP (88\% of ancestral allele A), c.3113A >G rs16941 SNP (84\% of ancestral allele
T), c.3548A $>\mathrm{G}$ rs2227945 SNP (96\% of ancestral allele T), c.7397C >T rs169547 SNP (91\% of ancestral allele C), c.7319A $>G$ rs 4986860 SNP ( $96 \%$ of ancestral allele A), c.4837A $>\mathrm{G}$ rs1799966 SNP (77\% of ancestral allele T). However, the rs16942 SNP, 22\% of the alleles found in the African population studies, are ancestral alleles (C).

Mutations in several other genes remain to be identified, even if AKT1, TP53 and PTEN, ATM variants have been associated with hereditary breast cancer [38]. Further work is required and include additional analyses of these genes known to be involved in breast cancer. Also, some studies reported the whole spectrum of hereditary breast cancer is not summarized by only BRCA1 and BRCA2 mutations [39-43]. A study in a Japanese population free of $B R C A$ mutation revealed some large deletions in RAD51C [43]. So RAD51C, RAD51D or even PALB2 could be explored. Esteller et al., have found a high rate of loss of heterozygosity at the BRCA1 locus that accounts for the vast majority of $B R C A 1$ biallelic inactivation, in most tumors from BRCA1 families [41]. Therefore, another perspective could focus on epigenetics in tumors.

\section{Conclusions}

This study describe some variants found in BRCA1 and BRCA2 genes, in young women with nodules and suspected breast cancer in Burkina Faso. Breast cancer in Burkina Faso can have a genetic basis because some of variants that we identified may be predisposing, but there is need to have a large number of specimen and more specific genetic testing to confirm if this can be considered as conferring an increased risk for breast cancer in this West African population.

Acknowledgement: We thank Mrs Pietro ANNIGONI, the St'Orsola Unit of Medical Genetics, University of Bologna, Italy, European Molecular Biology Organization (EMBO), and the Biomolecular Research Center Pietro Annigoni (CERBA/Labiogene) of Ouagadougou, Burkina Faso.

Conflict of interest: Authors state no conflict of interest.

\section{References}

1. IARC IAfRoC. Cancer in Africa: epidemiology and prevention. IARC Sci Publ. 2003;153:1-414.

2. Vorobiof DA, Sitas F, Vorobiof G. Breast cancer incidence in South Africa. J Clin Oncol. 2001;18 Suppl1:125-7. 
3. Couch FJ, DeShano ML, Blackwood MA, Calzone K, Stopfer J, Campeau L, et al. BRCA1 mutations in women attending clinics that evaluate the risk of breast cancer. $\mathrm{N}$ Engl J Med. 1997;336:1409-15.

4. Milne RL, Antoniou AC. Genetic modifiers of cancer risk for BRCA1 and BRCA2 mutation carriers. Ann Oncol. 2011;22 Suppl 1:11-7.

5. Nomura SJ, Dash C, Rosenberg L, Palmer J, Adams-Campbell LL. Sedentary time and breast cancer incidence in African American women. Cancer causes control : CCC. 2016;27:123952.

6. Greene MH. Genetics of breast cancer. Mayo Clin proc. 1997;72:54-65.

7. WHO. Cancer Country Profiles. World Health Organization. 2014; http://www.who.int/cancer/country-profiles/bfa_ en.pdf?ua=1.

8. Bambara HA, Zoure AA, Sawadogo AY, Ouattara AK, Ouedraogo NLM, Traore SS, et al. Breast cancer: descriptive profile of 80 women attending breast cancer care in the Department of General and Digestive Surgery of CHU-YO. Pan Afr Med. 2017;28:314.

9. Zoure AA, Bambara AH, Sawadogo AY, Ouattara AK, Ouedraogo $M$, Traore SS, et al. Multiparity and Breast Cancer Risk Factor among Women in Burkina Faso. Asian Pac J Cancer Prev. 2016;17:5095-9.

10. Bethea TN, Rosenberg L, Castro-Webb N, Lunetta KL, Sucheston-Campbell LE, Ruiz-Narvaez EA, et al. Family History of Cancer in Relation to Breast Cancer Subtypes in African American Women. Cancer Epidemiol Biomarkers Prev. 2016;25:366-73.

11. Miki Y, Swensen J, Shattuck-Eidens D, Futreal PA, Harshman K, Tavtigian S, et al. A strong candidate for the breast and ovarian cancer susceptibility gene BRCA1. Science. 1994;266:66-71.

12. Abdulrahman GO, Jr., Rahman GA. Epidemiology of breast cancer in europe and Africa. J Cancer Epidemiol. 2012;2012:915610.

13. Fregene A, Newman LA. Breast cancer in sub-Saharan Africa: how does it relate to breast cancer in African-American women? Cancer. 2005;103:1540-50.

14. Newman LA. Breast cancer in African-American women. Oncologist. 2005;10:1-14.

15. Samson ME, Porter NG, Hurley DM, Adams SA, Eberth JM. Disparities in Breast Cancer Incidence, Mortality, and Quality of Care among African American and European American Women in South Carolina. South Med J. 2016;109:24-30.

16. Futreal PA, Liu Q, Shattuck-Eidens D, Cochran C, Harshman $\mathrm{K}$, Tavtigian S, et al. BRCA1 mutations in primary breast and ovarian carcinomas. Science. 1994;266:120-2.

17. Wooster R, Neuhausen SL, Mangion J, Quirk Y, Ford D, Collins $\mathrm{N}$, et al. Localization of a breast cancer susceptibility gene, BRCA2, to chromosome 13q12-13. Science. 1994;265:2088-90.

18. Mote PA, Leary JA, Avery KA, Sandelin K, Chenevix-Trench $G$, Kirk JA, et al. Germ-line mutations in BRCA1 or BRCA2 in the normal breast are associated with altered expression of estrogen-responsive proteins and the predominance of progesterone receptor A. Genes chromosomes cancer. 2004:39:236-48.

19. Whittemore AS, Gong G, Itnyre J. Prevalence and contribution of BRCA1 mutations in breast cancer and ovarian cancer: results from three U.S. population-based case-control studies of ovarian cancer. Am J Hum Genet. 1997;60:496-504.

20. Rebbeck TR, Mitra N, Wan F, Sinilnikova OM, Healey S, McGuffog L, et al. Association of type and location of BRCA1 and BRCA2 mutations with risk of breast and ovarian cancer. JAMA. 2015;313:1347-61.

21. Zoure AA, Slaoui M, Bambara HA, Sawadogo AY, Compaoré TR, Ouédraogo NLM, et al. BRCA1 c.68_69delAG (exon2), c.181T>G (exon5), c.798_799delTT and 943ins10 (exon11) mutations in Burkina Faso. J Public Health Afr 2018;9:663.

22. Hartge P, Struewing JP, Wacholder S, Brody LC, Tucker MA. The prevalence of common BRCA1 and BRCA2 mutations among Ashkenazi Jews. Am J Hum Genet. 1999;64:963-70.

23. Thompson D, Easton D, Breast Cancer Linkage C. Variation in BRCA1 cancer risks by mutation position. Cancer Epidemiol Biomarkers Prev. 2002;11:329-36.

24. Basham VM, Lipscombe JM, Ward JM, Gayther SA, Ponder BA, Easton DF, et al. BRCA1 and BRCA2 mutations in a populationbased study of male breast cancer. Breast Cancer Res. 2002;4:R2.

25. Lawrenson K, Kar S, McCue K, Kuchenbaeker K, Michailidou $\mathrm{K}$, Tyrer J, et al. Functional mechanisms underlying pleiotropic risk alleles at the 19p13.1 breast-ovarian cancer susceptibility locus. Nat Commun. 2016;7:12675.

26. Bogorad RL, Courtillot C, Mestayer C, Bernichtein S, Harutyunyan L, Jomain JB, et al. Identification of a gainof-function mutation of the prolactin receptor in women with benign breast tumors. Proc Natl Acad Sci U S A. 2008;105:14533-8.

27. Jackson M, Marks L, May GHW, Wilson JB. The genetic basis of disease. Essays Biochem. 2018 ;62(5):643-723.

28. Adjiri A. DNA Mutations May Not Be the Cause of Cancer. Oncol Ther. 2017;5(1):85-101.

29. Zhou X, Han S, Wang S, Chen X, Dong J, Shi X, et al. Polymorphisms in HPV E6/E7 protein interacted genes and risk of cervical cancer in Chinese women: a case-control analysis. Gynecol Oncol. 2009;114:327-31.

30. Mefford HC, Baumbach L, Panguluri RC, Whitfield-Broome C, Szabo C, Smith S, et al. Evidence for a BRCA1 founder mutation in families of West African ancestry. Am J Hum Genet. 1999;65:575-8

31. Karami F, Mehdipour P. A comprehensive focus on global spectrum of BRCA1 and BRCA2 mutations in breast cancer. Biomed Res Int. 2013;2013:928562.

32. Peterlongo P, Chang-Claude J, Moysich KB, Rudolph A, Schmutzler RK, Simard J, et al. Candidate genetic modifiers for breast and ovarian cancer risk in BRCA1 and BRCA2 mutation carriers. Cancer Epidemiol Biomarkers Prev. 2015;24:308-16.

33. Zhang B, Fackenthal JD, Niu Q, Huo D, Sveen WE, DeMarco T, et al. Evidence for an ancient BRCA1 mutation in breast cancer patients of Yoruban ancestry. Fam Cancer. 2009;8:15-22.

34. Uhrhammer N, Abdelouahab A, Lafarge L, Feillel V, Ben Dib A, Bignon YJ. BRCA1 mutations in Algerian breast cancer patients: high frequency in young, sporadic cases. Int J Med Sci. 2008;5(4):197-202.

35. Amankwaa-Frempong E, Yeboah FA, Nguah SB, Newman LA. Breast Cancer Genetic Testing Among African Patients With Breast Cancer: Deoxyribonucleic Acid Extraction From Tumor Tissue and International Multidisciplinary Partnerships. JAMA Surg. 2017;152(8):800-801. 
36. Zeng C, Guo X, Long J, Kuchenbaecker KB, Droit A, Michailidou $\mathrm{K}$, et al. Identification of independent association signals and putative functional variants for breast cancer risk through fine-scale mapping of the 12p11 locus. Breast cancer res. 2016;18(1):64.

37. Szabo $\mathrm{Cl}$, King MC. Population genetics of BRCA1 and BRCA2. Am J Hum Genet. 1997;60:1013-20.

38. Boyault S, Drouet Y, Navarro C, Bachelot T, Lasset C, Treilleux I, et al. Mutational characterization of individual breast tumors: TP53 and PI3K pathway genes are frequently and distinctively mutated in different subtypes. Breast Cancer Res Treat. 2012;132:29-39.

39. Bartke T, Borgel J, DiMaggio PA. Proteomics in epigenetics: new perspectives for cancer research. Brief Funct Genomics. 2013;12:205-18.

40. Bosviel R, Durif J, Dechelotte P, Bignon YJ, Bernard-Gallon D. Epigenetic modulation of BRCA1 and BRCA2 gene expression by equol in breast cancer cell lines. Br J Nutr. 2012;108:1187-93.

41. Esteller M, Fraga MF, Guo M, Garcia-Foncillas J, Hedenfalk I, Godwin AK, et al. DNA methylation patterns in hereditary human cancers mimic sporadic tumorigenesis. Hum Mol Genet. 2001;10:3001-7.

42. Karsli-Ceppioglu S, Dagdemir A, Judes G, Lebert A, PenaultLlorca F, Bignon YJ, et al. The Epigenetic Landscape of Promoter Genome-wide Analysis in Breast Cancer. Sci Rep. 2017;7:6597.

43. Sato K, Koyasu M, Nomura S, Sato Y, Kita M, Ashihara Y, et al. Mutation status of RAD51C, PALB2 and BRIP1 in 100 Japanese familial breast cancer cases without BRCA1 and BRCA2 mutations. Cancer Sci. 2017;108(11):2287-2294. 